\title{
Libérer les images : Compte-rendu critique de Horst Bredekamp, Théorie de l'acte d'image, la découverte, 2015
}

Thomas Golsenne et Chloé Maillet

\section{OpenEdition}

\section{Journals}

Édition électronique

URL : http://journals.openedition.org/imagesrevues/4528

DOI : 10.4000/imagesrevues.4528

ISSN : 1778-3801

Éditeur :

Centre d'Histoire et Théorie des Arts, Groupe d'Anthropologie Historique de l'Occident Médiéval Laboratoire d'Anthropologie Sociale, UMR 8210 Anthropologie et Histoire des Mondes Antiques

\section{Référence électronique}

Thomas Golsenne et Chloé Maillet, « Libérer les images : Compte-rendu critique de Horst Bredekamp Théorie de l'acte d'image, la découverte, 2015 », Images Re-vues [En ligne], Hors-série 6 | 2018, mis en ligne le 06 août 2018, consulté le 30 janvier 2021. URL : http://journals.openedition.org/imagesrevues/ 4528 ; DOI : https://doi.org/10.4000/imagesrevues.4528

Ce document a été généré automatiquement le 30 janvier 2021.

\section{(c) (1) 8}

Images Re-vues est mise à disposition selon les termes de la Licence Creative Commons Attribution Pas d'Utilisation Commerciale 4.0 International. 


\section{Libérer les images : Compte-rendu critique de Horst Bredekamp, Théorie de l'acte d'image, la découverte, 2015}

Thomas Golsenne et Chloé Maillet

Théorie de l'acte d'image est issu d'une série de conférences données à Francfort en 2007 par l'historien de l'art allemand, célèbre en France pour ses livres analysant la dimension esthétique des sciences naturelles et de la philosophie (Les Coraux de Darwin, Stratégies visuelles de Thomas Hobbes ${ }^{1}$ ), mais aussi auteur de nombreux ouvrages sur l'art de la Renaissance, l'iconoclasme, et les nouvelles théories de l'image pas encore traduits en français².

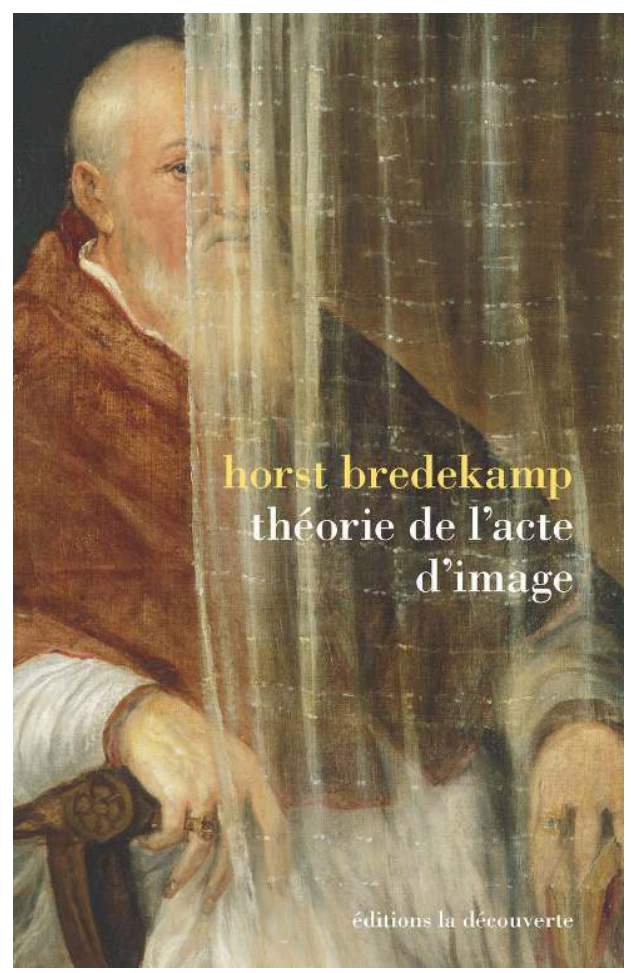


Fig. 1

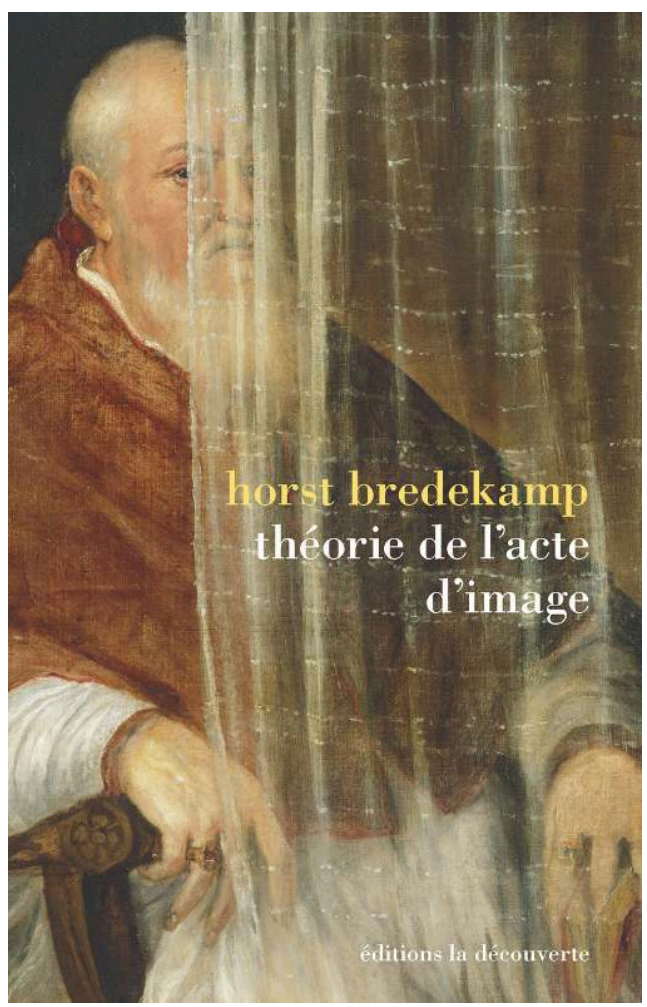

Horst Bredekamp, Théorie de l'acte d'image, Paris, Éd. la découverte, 2017

Dans ce livre ambitieux, Bredekamp apporte sa contribution à l'anthropologie des images à l'allemande (Bildanthropologie), dont plusieurs grands auteurs ont apporté des propositions majeures (Hans Belting, Gottfried Boehm notamment ${ }^{3}$ ), mais s'inscrit dans un cadre de réflexion plus large qui concerne l'action des images, leur "pouvoir " (David Freedberg), leur " agentivité » (Alfred Gell), leur "volonté » (W. Mitchell) ou leur «performance» (Bartholeyns, Dierkens et Golsenne)4. Toutes ces recherches partagent un rejet de l'histoire de l'art limitée à l'étude des œuvres conservées dans les musées, les analyses qui se cantonnent à l'histoire stylistique ou se bornent à interpréter leur signification. Mais de grandes différences les distinguent, à la fois sur le plan des images qu'ils privilégient et sur celui de la théorie qu'ils élaborent. Sur ces deux points, la position de Bredekamp est originale : c'est le seul à confronter images artistiques et images scientifiques (de ce point de vue, son ouvrage apparaît comme une synthèse de toute son œuvre) ; et sa théorie de l'image est élaborée à partir de (et contre) les théories de la performativité du langage. Mais, au-delà du projet original de l'auteur, son positionnement théorique permet aussi de dégager une orientation politique claire pour les historien'ne-s des images. Bredekamp l'annonce d'emblée : une des raisons majeures qui explique que le rôle social des images demande une réponse théorique, c'est la " portée politique des images » (9), qui ne se réduit pas à leur rôle traditionnel de "représentation du pouvoir", mais avance au contraire que «les images ont évolué jusqu'à devenir des armes de premier choix.» (10) Pourquoi la destruction des bouddhas géants de Bâmiyân a-t-elle choquée les sociétés occidentales ? Pourquoi la destruction du World Trade Center est-elle devenue l'image la plus partagée de l'histoire ? Pourquoi douze caricatures danoises ont-elles provoqué une crise mondiale et la mort de centaines de personnes ? La réponse de Bredekamp ne 
s'appuie ni sur une explication psychologique généralisante (qui chercherait dans les mécanismes neurologiques la raison du pouvoir des images), ni sur une philosophie du simulacre (qui expliquerait que le réel a disparu, que tout est devenu image), ni sur une anthropologie culturaliste (qui justifierait les réactions violentes face aux images de certaines populations, en raison de leurs croyances propres). Elle s'appuie sur « une phénoménologie de l'image active » (17), à savoir une analyse du fonctionnement des images, sur leur mécanisme interne en tant qu'images. C'est parce que, dans les cas pris en exemple précédemment, les images agissent sur le mode de la substitution que leur destruction apparait aussi violente : elles remplacent des êtres humains. On peut tuer pour des images, on peut tuer des images, on peut tuer des humains pour en faire des images. Bredekamp l'affirme clairement :

Les images peuvent être détruites à la place des personnes, ou comme si elles étaient des personnes : le principe même de l'agression iconoclaste. Toutes ces opérations sont régies par une pratique substitutive qui lorsqu'elle n'est pas comprise en tant que telle, peut conduire à ce que l'œuvre soit attaquée. (197)

3 Comme on le voit, il s'agit bien, pour l'auteur, d'un problème de connaissance. Mieux comprendre comment fonctionnent les images n'a pas qu'un intérêt scientifique : c'est aussi, et avant tout, un enjeu politique. Si l'on veut agir sur le monde et œuvrer à résoudre les conflits terribles qui déchirent l'humanité, il est urgent de comprendre que les images sont soumises à un régime de fonctionnement qui les transforme en armes. Autrement dit, il faut non seulement libérer les peuples prisonniers des chefs de guerre, mais aussi libérer les images elles-mêmes. Bredekamp ajoute en effet :

Les images doivent se voir reconnaitre un droit à l'existence afin de pouvoir venir à la rencontre du moi dans leur libre autonomie et non comme des substituts de ce moi. Telle est la raison la plus actuelle et la plus pressante d'élaborer une théorie de l'acte d'image politique, se situant entre ces pôles extrêmes de l'échange du corps et de l'image [...]. (197)

4 Il serait désormais temps de distinguer les images des personnes, de les tuer pour qu'elles ne puissent plus être considérées comme des humains, afin que l'on cesse d'en faire une motivation pour des meurtres ou des actes de vandalisme terribles, dont nous avons eu depuis les années 2000 des démonstrations pour le moins tragiques.

5 Ce constat provient de l'actualité la plus pressante, comme le souligne l'auteur, mais la déconstruction de ce système d'" images substitutives " provient de l'analyse d'une histoire des images de grande ampleur, en nous invitant à relire une bonne partie de l'histoire de l'art occidental. Pour bien comprendre que Bredekamp ne prend pas l'action des images pour quelque chose de seulement symbolique, et que leur «vie", leur « mort » ou leur capacité à blesser ou à être blessées n'a pas une portée seulement métaphorique, il faut en revenir d'abord au contenu théorique de l'ouvrage.

Dans le chapitre 1, qui entame l'analyse de la «vie» des images, Bredekamp définit d'abord cette dernière comme Gestaltung, formation, mise en forme par l'homme de la matière naturelle. Cette définition exclut la notion de ressemblance du concept d'image car elle ne se limite par aux œuvres dites figuratives, mais inclut toute production d'artefact, et même toute trace de faire humain. S'il fait référence à l'acte d'image déjà évoqué par Henri Lefèbvre dans sa Critique de la vie quotidienne (1961), Bredekamp ne mentionne pas le courant plus récent de l'anthropologie des objets ou de la culture matérielle et les études qui montrent comment les objets, et pas seulement les images, sont considérés comme vivants ou animés. Ce faisant, Bredekamp se rapproche de la théorie de l'anthropologue Alfred Gell5, présent dans sa bibliographie, mais qu'il ne cite 
pas explicitement dans le texte : Gell étudie en effet toutes les relations sociales qui passent par l'intermédiaire d'objets, lesquels sont alors dotés d'agentivité. Les images ne sont qu'un cas particulier dans sa "théorie anthropologique de l'art ». Bredekamp inverse la proposition : les œuvres d'art (et même les figurations) ne sont qu'un cas particulier de sa théorie des images.

7 Ainsi, cette définition très large de l'image comme formation permet à Bredekamp de remonter jusqu'aux bifaces : le biface est une image dans la mesure où il atteste l'activité de l'homme sur la matière, où il montre une volonté d'organisation de la matière. Ceci a pour conséquence de faire de l'image un critère de l'humanité : "l'homme est celui qui se montre capable de transformer des formations de la nature en images et de caractériser celles-ci comme une sphère à part entière [...] » (20-21) et de rapprocher image et technique. En cela, Bredekamp rejoint la définition de l'image simulacrum de Leon Battista Alberti, qui décrivait comme image n'importe quel objet de la nature montrant la trace d'un minimum de travail humain ; en s'appuyant également sur la culture chinoise des Gonshi (rochers de lettré), qui consistait à placer sur un socle sculpté des pierres à penser, sculptées par l'eau et le vent et parfois à peine corrigées par l'homme. Bredekamp affirme ainsi la nécessité de produire une théorie de l'acte d'image qui prenne en compte toutes ses dimensions, visuelles, tactiles, auditives, pour les mettre à l'épreuve (49). Que toute image soit le résultat de l'action humaine sur la matière inscrit dans un fondement paléoanthropologique le lien consubstantiel entre l'image et l'humain.

8 C'est également dans cette mesure que Bredekamp peut comparer l'image au langage, dans la perspective ouverte par John Austin sur les «actes de parole »: dire, c'est faire, l'équation du linguiste qui a lancé le champ des études sur la performativité du langage, suppose d'analyser les conditions d'énonciation, le point de vue du producteur de parole ${ }^{6}$. La phrase "Je vous marie » n'a par exemple aucun effet si elle n'est pas prononcée par le maire ou le prêtre, dans des circonstances ritualisées particulières.

Mais l'auteur laisse bien vite de côté le champ de la production des images pour s'intéresser à celui de leurs effets. Si l'image est le résultat d'une action humaine sur la matière, elle agit aussi sur les humains qui la regardent. Sa théorie vise à saisir «ce pouvoir [de l'image] qui lui permet, dans la contemplation ou l'effleurement, de passer de la latence à l'influence visible sur la sensation, la pensée et l'action. Ce questionnement suppose de comprendre l'acte d'image comme un effet sur le ressentir, le penser et l'agir, qui se constitue à partir de la force de l'image et de l'interaction avec celui qui regarde, qui contemple, effleure, et écoute aussi. » (44-45) Ainsi, plutôt que de transposer les catégories d'actes de langage répertoriés par Austin, Searle ou d'autres auteurs plus récents, au domaine des images, Bredekamp accomplit une inflexion décisive : sa théorie «ne met pas l'“image" à la place des mots, mais à la place du locuteur. » (44)

10 Autrement dit, c'est moins dans l'énonciation qu'il faut chercher la puissance des images que dans leur énoncé. Pour le comprendre, Bredekamp part de Platon (28-34). À première vue, on ne voit pas comment le philosophe grec, connu pour son mépris des images et de tout ce qui relève de la mimesis, peut servir de base à une théorie de l'acte d'image. Mais l'historien de l'art montre que le point de vue platonicien est plus complexe. Certes, d'un côté, Platon exprime un rejet des images et de l'imitation, qu'il accuse de détourner des idées, comme autant d'illusions qui éloignent de la vérité. Mais, ailleurs, l'image peut servir de modèle qui aide à la connaissance de phénomènes 
complexes (en astronomie), à rendre intelligible une idée (la forme de la cité idéale), qui fixe un idéal d'ordre moral et esthétique (chez les Égyptiens). Bredekamp résume la position paradoxale de Platon ainsi :

Les images, selon Platon, agissent en tant que fondements de la pensée et de l'agir mais, d'un autre côté, elles dissimulent la vérité. Platon n'était hostile qu'aux images qu'il considérait comme une menace pour la communauté, alors qu'il a défendu et salué les images qu'il reconnaissait comme un facteur de civilisation.

(34)

11 L'entreprise de Bredekamp ne consistera pas à trier les «bonnes » et les «mauvaises » images, à établir une "police des images", car ce serait là adopter une position résolument opposée à la modernité. L'historien de l'art ne cherchera pas non plus à faire de l'image un tremplin qui permette de passer du sensible à l'intelligible, la débarrassant au passage de tout ce que le langage et l'entendement ne peuvent absorber, à la manière de Kant et des néo-kantiens Cassirer et Panofsky. Il cherchera plutôt à expliquer pourquoi les images peuvent produire désir et haine, connaissance et mensonge, consensus et dissensus.

Dans le chapitre 2, Bredekamp évoque les « œuvres-énoncés », les images parlantes par les inscriptions qui leurs sont apposées. Il commente certains vases grecs, qui portent parfois des inscriptions à la première personne qui les font parler. Or, bon nombre de ces inscriptions concernent le producteur du vase : «Untel a fait cela», «Untel m'a fait " (hoc fecit plus distancié en latin, devenu parfois me fecit). Dans ce cas, l'image ne parle pas pour paraître non faite par un humain, pour paraître un être vivant et non un artefact ; la relation est plutôt de l'enfant à son père, une relation d'engendrement, de procréation. Il cite et fameux "Gislebert a fait cela" (Gislebertus hoc fecit) de la cathédrale d'Autun et la signature de l'autel de Saint-Sernin à Toulouse : "Bernard Gilduin m'a fait », (64). La tradition, à laquelle fait référence Bredekamp voulait que Gislebert soit l'artiste, orgueilleusement placé sous les pieds du christ en majesté. Mais les dernières recherches soutiennent plutôt que Gislebert serait un commanditaire?

En prenant en exemple les œuvres de Niki de Saint-Phalle dont Tu es moi et les Tirs, Bredekamp affirme que cette logique va jusqu'au point paradoxal où le créateur et l'œuvre s'échangent, se substituent, mais dans la destruction. De son côté, Van Eyck, dans ses inscriptions "Als ich kan " et "Van Eyck me fecit ", porte la contradiction de deux « Je » : le je de l'auteur et celui de l'œuvre.

Ces différentes positions montrent que les images sont dotées d'un pouvoir intrinsèque qui inquiète leurs auteurs et spectateurs, qu'ils cherchent à résorber ou à expliquer par des phénomènes extérieurs (comme le pouvoir divin). Or, affirme Bredekamp, le pouvoir des images a sa cause en elles-mêmes car elles montrent « une sphère refoulée du rapport des hommes au monde organisé » (91). Bredekamp en revient finalement à Platon et au besoin de contrôler les images dont le pouvoir serait dangereux. Le thème du refoulement renvoie aussi à Freedberg, qui expliquait que le pouvoir des images a été refoulé par la culture européenne de l'art. Bredekamp ne cite pas le livre de Jack Goody, La peur des représentations, qui allait pourtant dans son sens en essayant de dépasser le point de vue eurocentrique. Mais il est vrai que la théorie de l'image de Bredekamp se distingue d'une théorie de la représentation, puisque tout artefact est image pour lui. Ainsi les épées qui sont signées à la première personne sont-elles aussi vivantes que les peintures. Bredekamp ne s'attarde pas sur la question de savoir pourquoi les épées sont signées, et pas d'autres objets : mais ce sont des objets de prestige et, de plus, leur utilisation les met en mouvement. Or il est commun dans les 
romans de chevalerie que des épées magiques soient dotées d'une force autonome : ce n'est pas le bras qui les bouge, mais elles qui se meuvent toutes seules.

Fig. 2

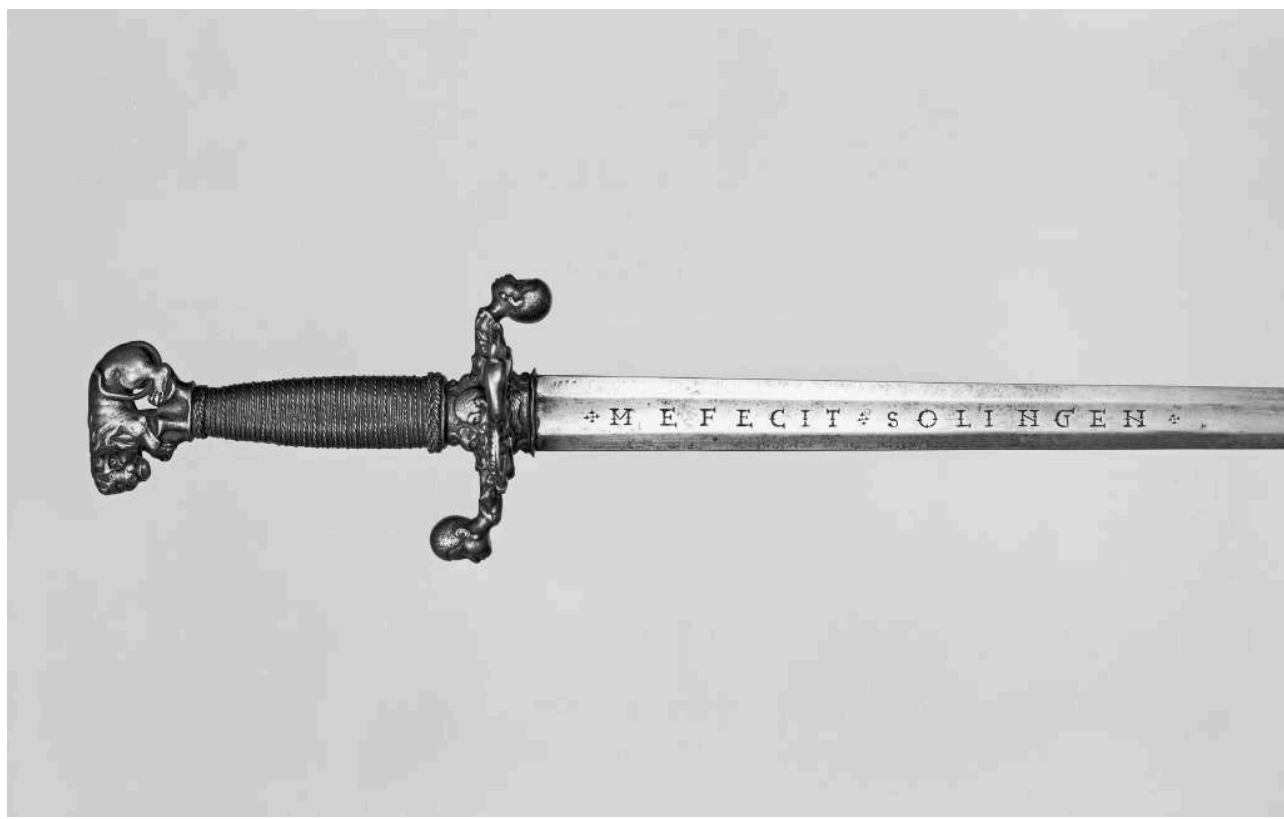

Épée légère pommeau attr. Gottfried Leygebe, lame signée Meves Berns, Solingen, v. 1650 102,5 cm. Baltimore Walters Art Gallery

Dans le chapitre 3, Bredekamp commence à catégoriser les actes d'image : ils peuvent être schématique, substitutif ou intrinsèque. Il ne s'agit pas là de types d'images : ainsi, la même image peut sans doute accomplir plusieurs actions. Par exemple, un tableau vivant de la Passion réalise l'acte schématique (les corps deviennent des images) et l'acte substitutif (les acteurs jouent le Christ, les Romains...). Le portrait de Baldassare Castiglione par Raphaël, tel que Castiglione le décrit (à travers une fausse lettre de son épouse), agit comme schéma (il est vivant) et comme substitut (il prend la place du mari absent). Cependant, certaines images possèdent une inclination, comme nous allons le comprendre, pour certains actes d'image. Afin d'expliciter ces concepts, Bredekamp va choisir des exemples très variés. Il commence par les actes d'images schématiques, du grec schema, contour, modèle, et qui sert à Platon pour expliquer comment les images sont des imitations. Pour le philosophe grec, les schemata « sont les modèles des enchaînements de mouvements stéréotypés au cours desquels les corps deviennent images. » (32), par exemple dans les circonvolutions des astres cosmiques, dans les gestes codifiés de la danse. Modernisant cette théorie par l'entremise de la "soma-esthétique " de Richard Susterman ${ }^{8}$, Bredekamp définit l'acte d'image schématique comme la façon dont l'image fait du corps le «fondement [...] qui permet aux humains de reconnaître les autres, eux-mêmes et le monde, et de se comporter dans cet environnement. » (94)

Pour Bredekamp, c'est par empathie que les images schématiques sont activées, c'est-àdire deviennent vivantes : une projection psychologique. Il trahit ici son attachement à l'esthétique allemande. Mais l'important n'est pas là : il réside dans le fait qu'à ses yeux les images schématiques estompent la frontière entre le vivant et l'inerte, entre la représentation et son prototype. 
Les tableaux vivants sont ainsi des exemples d'images schématiques : ce n'est pas parce que les acteurs arrêtent de bouger pour imiter la peinture, mais plutôt parce qu'on ne peut plus distinguer ce qui est peint de ce qui est vivant. Une œuvre d'art contemporain exemplaire pour l'auteur est Singing Sculpture (1970) de Gilbert et George, dans la mesure où le but de cette œuvre est d'abolir la distinction entre l'humain, la sculpture et le vivant.

Fig. 3

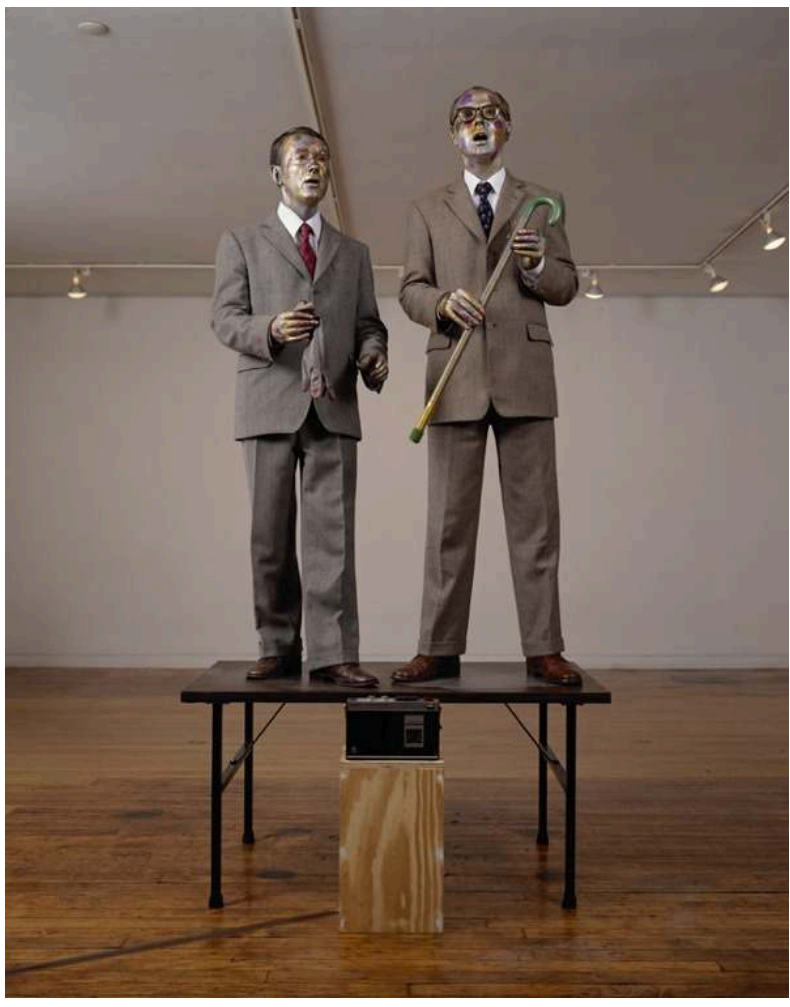

Gilbert and George, The Singing Sculpture, 1970, Londres, Nigel Greenwood Galerie

Bredekamp a raison de citer un précédent bien plus ancien à la living sculpture en même temps qu'à l'art de la performance : les Attitudes de Lady Hamilton, immortalisées entre autres par une peinture d'Élisabeth Vigée Lebrun. Visiblement assez peu familier des études d'histoire féministe, l'auteur affirme que c'est à la demande de son mari Lord Hamilton que la jeune femme prenait des poses dans les salons mondains comme une sculpture vivante, au point que ses « attitudes " plastiques et ses tenues spécifiques la rendirent très connue. C'est l'inverse qui arriva : Emma Hart était seule devenue performeuse de renom, modèle de plusieurs peintres, bien avant de rencontrer l'amateur d'antiquités et de belles femmes9. Mais ce sont bien les séances qu'il organisait pour elle dans sa villa (ainsi que sa liaison avec l'amiral Nelson) qui en firent une célébrité mondiale. 
Fig. 4

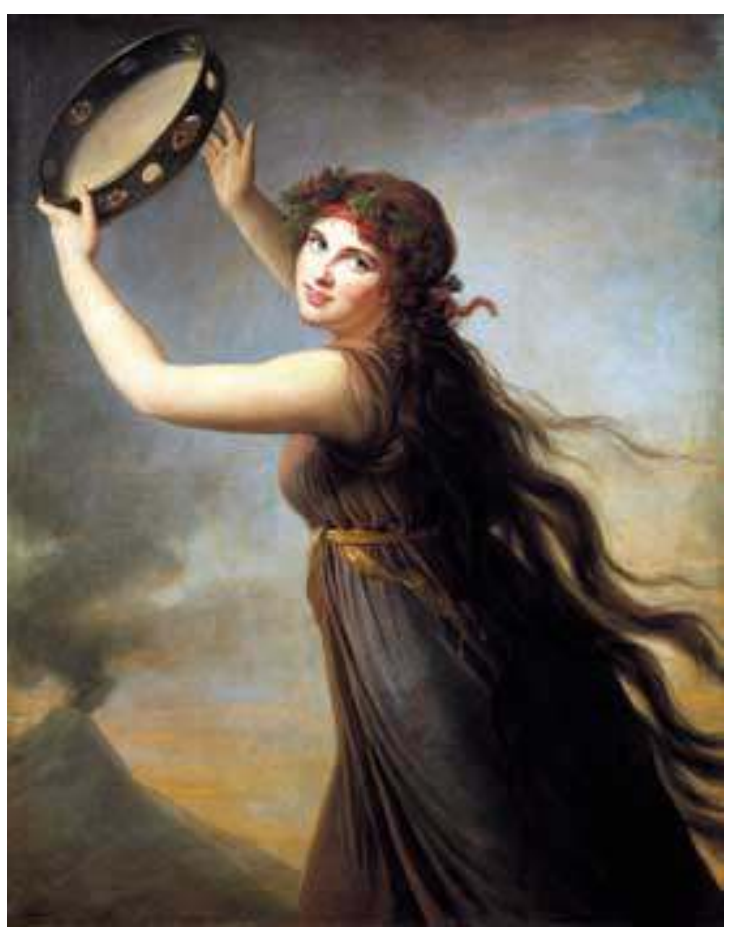

Élisabeth Vigée Lebrun, Lady Hamilton en bacchante, v. 1792, huile sur toile, 159 × 131,5 cm, Liverpool, Liverpool, National Museum, Lady Lever Collection

En réciproque au tableau vivant, l'automate est un artefact qui imite la vie. Les analyses de Bredekamp s'appuient sur des exemples historiques (un automate du XVI siècle, l'automate écrivant de Jaquet-Droz) et embrassent un large champ jusqu'à la sciencefiction. Un cas particulier boucle la boucle : la danse de robot de Michael Jackson, humain vivant qui imite l'automate qui imite l'humain.

Le chapitre continue logiquement avec les poupées et Pygmalion, exemple archétypal de l'animation de l'œuvre d'art. Sans redoubler le livre de Victor I. Stoichita sur le sujet $^{10}$, l'auteur insiste sur la réitération du motif par les surréalistes jouant à la poupée. Sur ce point, le livre aurait gagné à être étayé par les analyses anthropologiques de Gell et de ses continuateurs ${ }^{11}$, et aussi de prendre en charge la dimension genrée de cette mise en scène de l'homme créateur et de la femme créature. Pygmalion, Bellmer et les surréalistes, Jensen sont des hommes qui créent des poupées femmes, pour jouer à l'art et à l'amour. Ce n'est pas anecdotique car cela s'inscrit au cœur de la pensée européenne de la production comme procréation, rapport sexuel d'engendrement.

Reste la question de la nécessité de donner vie aux images :

Comme nous l'avons vu, les images ont été amenées à parler parce qu'elles sont muettes; elles ont été mises en scène à travers des corps car elles sont faites d'un matériau mort ; elles se sont vu donner un mouvement interne car elles sont immobiles; elles ont été pourvues d'émotions de façon artificielle parce qu'elles ne nourrissent pas elles-mêmes des sentiments. (157)

Mais cette explication, à bien la considérer, paraît un peu légère : si l'on a cru bon trouver des techniques d'animation des images parce qu'elles en manquaient, pourquoi avait-on besoin, en premier lieu, d'animer les images ? Il faut comprendre que leur mode d'action (la schématisation) est consubstantielle à leur aspect (l'anthropomorphisme). Ce faisant, l'auteur montre les limites anthropologiques de sa 
théorie, circonscrite à un cadre culturel et historique et européen, où les images anthropomorphes ont largement dominé le champ visuel. Bredekamp avait été invité à présenter son ouvrage lors de l'exposition Persona au musée du quai Branly, une exposition organisée par Anne Christine Taylor et Emmanuel Grimaud ${ }^{12}$. L'exposition avait justement pour but de montrer les formes variables de l'anthropomorphisme ou de l'abduction d'agentivité dans les artefacts, mais sans que cela passe obligatoirement par la mise en jeu du corps humain représenté et sans que cela soit forcément inquiétant.

Le chapitre 4 est consacré à l'acte d'image substitutif. «Dans la substitution, les corps sont traités comme des images et les images comme des corps. » (159) L'image prend la place de son modèle, le remplace. Dans l'acte d'image schématique, Bredekamp insiste sur la transformation du corps vivant en image et de l'image en corps vivant, mais sans forcément qu'il y ait substitution de l'un à l'autre. L'acte d'image substitutif n'est pas un acte de transformation, mais de représentation. L'image ne devient pas vivante, elle prend la place d'un être vivant. Cette catégorie d'action comprend évidemment un nombre d'images presque infini, puisqu'elle couvre toutes les formes de représentation figurative (les portraits et les icônes, les paysages et les natures mortes) et explique les actes de destruction ou de création d'images.

L'acte substitutif d'image a notamment pour application le champ religieux (présence de l'être incarné dans l'image), juridique (force de preuve de l'image) et politique (présence du souverain dans ses images). Ce dernier aspect est développé par Bredekamp à partir des pièces de monnaie (177) qu'il compare à l'acte illocutoire par lequel un bateau est baptisé ou un mariage est prononcé. Si le pouvoir du souverain est développé par la réitération de ses images, la communauté démocratique peut aussi être renforcée par des images qui la fédèrent, ainsi que le montrait le philosophe Whitehead à partir d'une analyse du drapeau, que Bredekamp reprend à son compte (181).

L'image a la faculté de performer le pouvoir. Comment ? Par sa présence elle s'impose comme vraie, répond Bredekamp, inspiré ici par le modèle chrétien de la Véronique plus que par Platon. Il développe l'exemple de l'image du Léviathan de Hobbes, figurée sur la couverture du livre éponyme du philosophe politique (179-181). 
Fig. 5

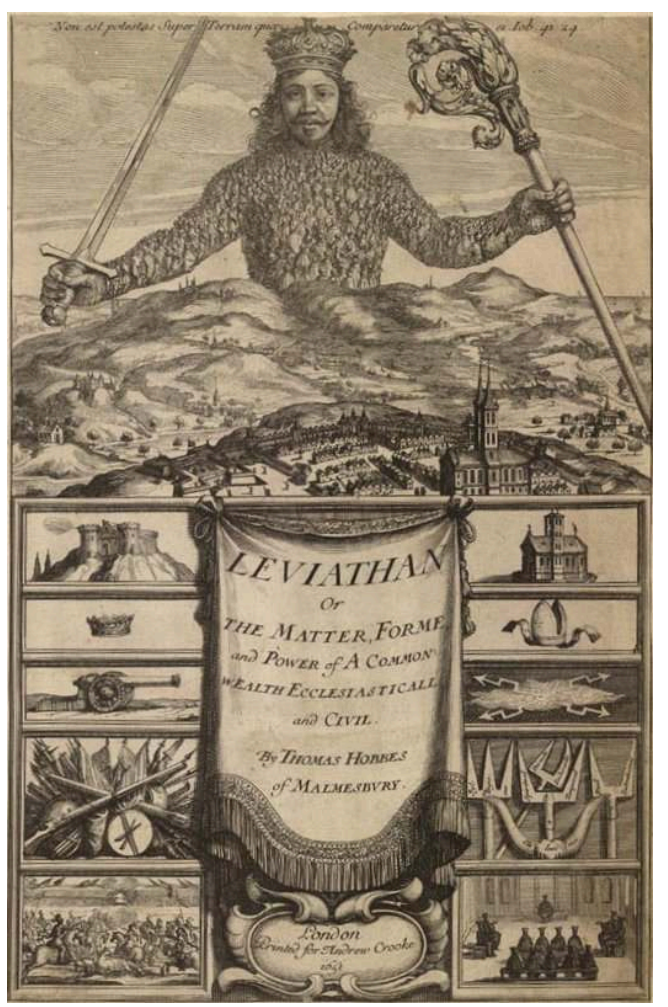

Thomas Hobbes, Leviathan, or the Matter, Forme, and Power of a Common Wealth Ecclesiasticall and Civil, Londres, 1651, frontispice attribué à Abraham Bosse

L'image de couverture, selon l'historien allemand, n'a pas que pour fonction de symboliser les idées contenues dans le livre, mais elle doit les rendre actives. Et ceci se produit dès qu'elle devient un signe, c'est-à-dire qu'on cherche à l'interpréter, qu'on réussit à la comprendre et qu'on en tire des conclusions pour agir. Mais comment fonctionne ce signe visuel, en quoi il se distingue du signe discursif, Bredekamp ne l'explique pas. Il faut ici supposer que signifier est un acte d'image - ce qui pourrait contribuer à estomper la spécificité de son approche eu égard à une iconologie plus classique. Le même problème s'est d'ailleurs déjà posé à propos des actes de discours ${ }^{13}$.

Les images infamantes que Bredekamp décrit (182) comme substituts aux condamnés à mort ne signifient pas seulement leur condamnation : elles l'effectuent sur un plan qu'on pourrait qualifier de symbolique si ce mot n'avait pas une connotation d'inefficacité aujourd'hui. Dans la logique magique de la pré-modernité européenne, pendre quelqu'un en image c'était comme le pendre lui. Ainsi, ce n'est pas parce qu'elle est un signe interprétable que l'image fonctionne : c'est parce qu'elle est présente, parce qu'elle est visible et qu'elle est vue. Étant vue, elle fait croire à l'existence de ce qu'elle représente ; la vérité de l'image de substitution n'est pas un pré-requis, c'est un effet de sens. Et s'il est vrai que l'on en a besoin dans les procédures judiciaires, pour témoigner ou prouver un fait, ce n'est pas parce que l'image délivrerait automatiquement la vérité ; c'est parce la construction des procédures de vérification détermine ce que représente l'image comme vraie. Ces procédures changeraient, la véracité de l'image serait peut-être remise en cause.

Bredekamp affirme que la photographie a mis un coup d'arrêt à la punition par l'image (185) en la faisant rentrer dans le domaine du droit de propriété : chacun devient 
propriétaire de son image, surtout les célébrités qui font de leur image un instrument de pouvoir. Ainsi les procès contre les paparazzi qui volent les images. Bredekamp voit une survivance de la punition par l'image dans les portraits robots et les avis de recherche où l'on diffuse l'image des dangereux criminels. On pourrait lui opposer que si les condamnations par l'image ont disparu, c'est parce que la justice est devenue moins visuelle, moins spectaculaire : on enferme les condamnés, on pend les prisonniers en secret, comme l'a montré Foucault ${ }^{14}$. Cela n'a donc peut-être pas à voir avec un changement ontologique de l'image (l'avènement de la photographie) mais un changement dans le droit et la justice.

À propos de l'iconoclasme, Bredekamp remarque qu'il fonctionne sur la substitution de l'image et du corps qu'on cherche à détruire. L'iconoclasme pourrait être évité si l'acte de substitution était enrayé. Il en appelle au respect des images : « les images doivent se voir reconnaître un droit à l'existence afin de pouvoir venir à la rencontre du moi dans leur libre autonomie et non comme des substituts de ce moi. » (197)

31 L'exemple donné par Bredekamp de la " guerre des images » contemporaine, entre talibans, al Qaida et autres islamistes terroristes, et les Américains et leurs alliés, renforce son argument que protéger les images, c'est aussi protéger les humains, car dans ces guerres « asymétriques » les deux sont interchangeables. Ainsi, avant, on tuait une image en substitution de la personne ; les terroristes tuent des personnes pour les transformer en images de terreur (213). Dans les images infamantes de la Renaissance, il y avait un décalage temporel entre l'exécution de la peinture et l'exécution de la personne ; à présent, c'est le meurtre qui devient l'image. Ce rapprochement de l'image et de l'acte renforce son efficacité. D'où la conclusion humaniste de Bredekamp :

Tous ces processus convergent du fait même que l'échange du corps et de l'image est partout utilisé comme arme de guerre, et bien qu'il ne soit pas couvert par la Convention de Genève. Tout cela montre la nécessité impérieuse de prendre au sérieux, dans une perspective historique, l'acte d'image substitutif, et de le comprendre, le désigner et de combattre son aspect destructeur. Les formes extrêmes de substitution font de la séparation de l'image et du corps un devoir si l'on veut maintenir vivant le programme des Lumières. (214)

Dans le chapitre 5, Bredekamp définit le troisième acte d'image qu'il appelle " intrinsèque ": l'image n'est pas anthropomorphisée pour devenir vivante, elle ne substitue pas à une personne ; elle agit par elle-même, en tant que forme. Ce type d'action de l'image repose sur les théories du regard et de l'imagination, qui postulent leur efficacité matérielle - ainsi des croyances au « mauvais œil » (219). 
Fig. 6

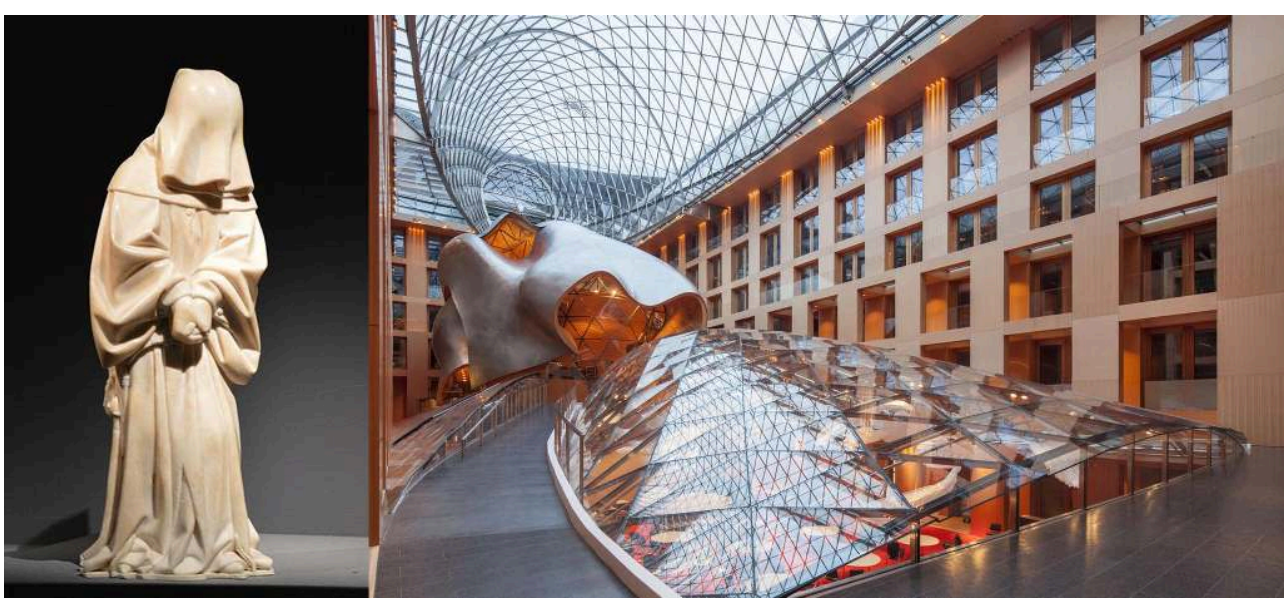

Claus Sluter et atelier, Pleurant du Tombeau de Jean sans peur, 1389-1406, albâtre, Dijon, Musée des beaux-arts / Frank Gehry, DZ Bank, 1996-2001, Berlin

Ce chapitre associe des exemples et des analyses tirés de l'histoire de l'art formaliste (par exemple, comment les capuchons de Claus Sluter ont marqué Frank Gehry qui s'en est servi pour l'auditorium de la Deutsche Bank, 262-264) et des analyses beaucoup plus originales qui remontent, encore une fois, aux origines européennes du problème. Ici il ne s'agit plus de partir de la Véronique, modèle de l'image substitutive, mais du pouvoir pétrifiant de la tête de Méduse et même de ses représentations, des scènes de Crucifixion figurées par les artistes des $\mathrm{XV}^{\mathrm{e}}-\mathrm{XVI}^{\mathrm{e}} \mathrm{s}$. comme Holbein qui font saigner le corps du Christ aussi bien que la surface du tableau (233). Le regard peut être frappé par n'importe quoi : il n'est plus besoin de voir un corps figuré pour être touché par l'acte intrinsèque de l'image. Le punctum de Roland Barthes, ce point mystérieux qui " poigne » le regardeur dans une photographie, et qui peut consister en n'importe quoi sur l'image, est là pour le rappeler (cité 232). Puisque ce n'est pas ce que l'image figure qui agit sur le spectateur, c'est l'image elle-même, dans sa matérialité, son opacité.

Bredekamp remonte le temps jusqu'aux performances des années 60 : Yves Klein, Yoko Ono, etc. (238) : quand le médium, c'est le corps de l'artiste, le sang devient sa matière première. Il revient ensuite à l'art de la Renaissance et notamment à la peinture vénitienne et à la faculté de Titien d'utiliser la couleur comme de la chair (246); à ce point, on retrouve des considérations bien connues en histoire de l'art sur la couleur comme animation ${ }^{15}$. Ce que cherche à définir ici Bredekamp, ce sont des propriétés matérielles de l'image qui produisent son animation ; pas étonnant dès lors qu'il aille jusqu'à la peinture abstraite et Lucio Fontana, dont il compare habilement les toiles fendues aux images médiévales de la plaie du Christ (242-243). Il aurait pu développer ce point en montrant que le crédo de l'autonomie de l'art, chez les modernistes, hérite d'une pensée magique où les images sont vivantes ; mais comme à ses yeux il s'agit d'actes d'images différents, il ne peut pas les ordonner en une séquence chronologique. Il importe pour lui de séparer ces différents actes. À la fin de ce chapitre, lorsqu'il évoque formules de pathos de Warburg $(272 \mathrm{sq})$ et qu'il dit qu'elles servent à introduire une distance entre l'image et le moi (c'est la fonction régulatrice des images d'art, selon Warburg, qui fait entrer l'humanité dans la civilisation et la dégage de la peur primitive des forces invisibles), il explique que si l'image possède une force intrinsèque, autonome du moi, dont la formule de pathos serait le paradigme, alors il faut se servir

Images Re-vues, Hors-série 6 | 2018 
de cette force comme antidote ou réponse à la force substitutive de l'image qui peut entraîner les humains dans un cycle de destruction (284-85).

La conclusion du livre fait se rencontrer Warburg et Darwin en passant par Roger Caillois. Warburg avait trouvé dans L'Expression des émotions chez l'homme et les animaux de Darwin un fondement de sa propre théorie des formules de pathos. Le naturaliste anglais pensait que les formes naturelles possédaient une force d'attraction variable, favorisant la reproduction sexuelle, et que les plus efficaces étaient renforcées par l'effet de la sélection naturelle. Il ne pouvait pas ne pas attribuer cette création surabondante de formes à un "dessein "; c'est pourquoi il pouvait les comparer à la peinture de Raphaël (290). Caillois va un peu plus loin encore, en qualifiant la formation des formes naturelles de véritables œuvres d'art. Bredekamp trouve encore chez Adolf Portmann, théoricien de l'élégance animale ou chez Stéphane Leduc, biophysicien du début du XX $\mathrm{XX}^{\mathrm{e}}$ siècle qui définissait l'origine de la vie comme un processus spontané de "devenir-image ", la confirmation de son idée principale : l'affinité du processus vital avec un acte d'image. On pourrait le dire autrement: la réalité est image et la vie est son acte.

Il pourrait sembler contradictoire de finir ainsi sur un dépassement des frontières modernes entre le naturel et le culturel, l'humain et l'animé, les sciences de l'art et les sciences de la nature, alors qu'au début de son livre, Bredekamp affirmait la spécificité humaine de la fabrication d'images. Mais cette contradiction n'est qu'apparente:

Toutes les énergies issues de l'activité visuelle de la nature [...] sont dans le domaine spécifique des artefacts érigées en principe propre de l'existence de ceux-ci. C'est pour cela que les images sont ressenties comme des entités actives autonomes, et qu'elles font la jonction entre monde inorganique et monde organique. (301)

En d'autres termes, les êtres vivants déploient une pluralité de formes, agissent comme des images, en vue d'une fin supérieure : la séduction en vue de la reproduction sexuelle et à terme, de la survie de l'espèce. Mais les artefacts, les œuvres d'art, si leur action fondamentale leur est intrinsèque, développent ce monde infini de formes sans d'autre fin que leur propre plaisir à exister.

En somme, on pourrait comprendre le livre de Bredekamp comme une théorie qui justifierait une déclaration universelle des droits de l'image : non seulement elles possèdent un droit à l'existence, affirme-t-il, mais elles ne devraient pas être utilisées à des fins de substitution (306). Son livre montre bien les dangers d'une telle utilisation. Le droit des images à exister pour elles-mêmes, à être libres et autonomes, à être vivantes, ouvre plutôt la voie à un cycle vertueux de créativité, où l'existence humaine retrouve le fondement biologique de la vie comme productrice d'images.

\section{NOTES}

1. Horst BREDEKAMP, Stratégies visuelles de Thomas Hobbes. Le Léviathan, archétype de l'État moderne [1999], trad. D. Modigliani, Paris, Éd. de la MSH, 2003 ; IDEM, Les coraux de Darwin. Premiers modèles 
de l'évolution et tradition de l'histoire naturelle [2003], trad. C. Joschke, Dijon, Les presses du réel, 2008.

2. Citons simplement Machines et cabinets de curiosité [1993], trad. N. Casanova, Paris, Diderot éd., 1997 ; Galileis denkende Hand. Form und Forschung um 1600, Berlin - Boston, de Gruyter, 2015.

3. Hans Belting, Pour une anthropologie des images [2001], trad. J. Torrent, Paris, Gallimard, 2004 ; Gottfried воєнм, Wie Bilder Sinn erzeugen. Die Macht des Zeigens, Berlin, 2007.

4. David FREEDBERG, Le pouvoir des images [1989], trad. Alix Girod, Paris, G. Monfort, 1998 ; Alfred GELL, L'art et ses agents. Une théorie anthropologique [1998], trad. O. et S. Renaut, Dijon, Les presses du réel, 2009 ; William J. T. MITCHELL, Que veulent les images? [2005], trad. M. Boidy, N. Cilins et S. Roth, Dijon, Les presses du réel, 2014 ; Gil BARTHOLEYNS, Alain DIERKENS et Thomas GOLSENNE (dir.), La performance des images, Bruxelles, Éd. de l'Université de Bruxelles, 2012.

5. Alfred GELL, L'art et ses agents, op. cit. Pour une relecture de ce texte appliquée à l'iconoclaste suffragette Mary Richardson, cf. Sophie MoIRoux, "L'image empreinte d'intentions. La "Vénus tailladée ». Considérations sur un acte d'iconoclasme ", Images Re-vues [En ligne], 2 | 2006, document 2, mis en ligne le 01 janvier 2006, URL : http://imagesrevues.revues.org/230.

6. John L. Austin, Quand dire c'est faire [1962], trad. G. Lane, Paris, Éditions du Seuil, 1970.

7. Linda SEIDEL, Legends in Limestone. Lazarus, Gislebertus, and the Cathedral of Autun, Chicago, The University of Chicago Press, 1999.

8. Richard shusterman, Conscience du corps. Pour une soma-esthétique, trad. N. Vieillescazes, Paris, L'Éclat, 2007.

9. Aileen RIBERo, The Art of Dress. Fashion in England and France 1750 to 1820, New Haven \& London, Yale University Press, 1995, p. 218-19.

10. Victor I. sтогснітA, l'Effet Pygmalion. Pour une anthropologie historique des simulacres, Genève, Droz, 2008.

11. En France, par exemple Agnès GIARD, Un désir d'humain. Les love doll au Japon, Paris, Les belles lettres, 2016.

12. Persona. Étrangement humain, Paris, Musée du quai Branly / Arles, Actes Sud, 2016.

13. Jean WIRTH, «Performativité de l'image ?", in La performance des images, op. cit., p. 125-35 et Irène ROSIER-CATACH, « Les mots et les images ", in ibid., p. 243-53.

14. Michel fOUCAULT, Surveiller et punir. Naissance de la prison, Paris, Gallimard, 1975.

15. Georges DIDI-HUBERMAN, La peinture incarnée, suivi de Le chef d'œuvre inconnu d'Honoré de Balzac, Paris, Éd. de Minuit, 1985.

\section{RÉSUMÉS}

Compte-rendu de Horst Bredekamp, Théorie de l'acte d'image : conférences Adorno, Francfort 2007, traduit par Frédéric Joly et Yves Sintomer, Paris, la Découverte, 2015, 376 p.

Review of Horst Bredekamp, Théorie de l'acte d'image : conférences Adorno, Francfort 2007, traduit par Frédéric Joly et Yves Sintomer, Paris, la Découverte, 2015, 376 p. 


\section{AUTEURS}

\section{THOMAS GOLSENNE}

Maître de conférences en histoire de l'art moderne et culture visuelle, Thomas Golsenne est membre du comité de rédaction d'Images Re-vues. Il a dirigé (avec G. Bartholeyns) La performance des images (Bruxelles, 2012) et publié Carlo Crivelli et le matérialisme mystique du Quattrocento (Rennes, 2016). Il est co-directeur du numéro Images émancipatrices.

\section{CHLOÉ MAILLET}

Chloé Maillet est docteure en anthropologie médiévale, artiste et professeure d'histoire de l'art à l'Ecole Supérieure des Beaux-Arts d'Angers. Elle a notamment publié La parenté hagiographique (XIIIe-XVe siècle) : d'après Jacques de Voragine et les manuscrits enluminés de la "Légende dorée" (c. 1260-1490), Turnhout, 2014 et prépare un ouvrage sur les figures transgenres au Moyen Âge. Elle est membre du comité de rédaction d'Images re-vues et co-directrice du hors-série Images émancipatrices. 